\title{
Educación alimentaria como estrategia para la reducción de la anemia en ocho regiones del Perú - 2020
}

\author{
Food education as a strategy to reduce anemia in eight regions of Peru- 2020
}

\author{
Rosa Elena Cruz ${ }^{1}$ \\ rosa.cruz@uwiener.edu.pe \\ Iván Carbajal ${ }^{2}$ ○ \\ ivancarbajalgomez@yahoo.es \\ Saby Mauricio ${ }^{\circledR}$ \\ saby.mauricio@uwiener.edu.pe \\ Michelle Lozada-Urbano ${ }^{3}$ ๑ \\ michelle.lozada@uwiener.edu.pe
}

Artículo recibido: 28/05/2021

Revisado por pares

Artículo aceptado: 24/01/2022

Artículo publicado: 25/02/2022

Autor de correspondencia

Michelle Lozada-Urbano,

michelle.lozada@uwiener.edu.pe

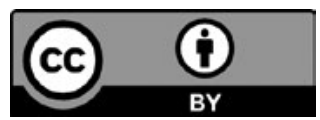

(CLos autores, 2022. Publicado por la Universidad Norbert Wiener (Lima, Perú)

Citar como: Cruz RE, Carbajal I, Mauricio S, Lozada-Urbano M. Educación alimentaria como estrategia para la reducción de la anemia en ocho regiones del Perú - 2020. Revista de Investigación (de la Universidad Norbert Wiener). 2022;11(1): a0003.

doi: https://doi.org/10.37768/unw.rinv.11.01.a0003

\begin{abstract}
Resumen
Introducción: En el Perú, aunque se observa una disminución de la anemia del 3,4\% en el año 2019, con relación al 2018, la prevalencia continúa desde hace más de una década, con cifras que la califican como un problema severo de salud pública, pues afecta a un promedio de 620000 niños a nivel nacional. La prevalencia de anemia en niños y niñas menores de 36 meses es más alta en la zona rural y en la sierra. A pesar de que Lima Metropolitana tiene una de las prevalencias más bajas del país (3 de cada 10 niños presentan anemia), concentra el mayor número de niños con anemia, con cifras similares al número de niños que obtenemos al sumar las cuatro o cinco regiones con mayor prevalencia en el país. Objetivo: Evaluar si la estrategia en educación alimentaria permite una reducción de la anemia en los niños de 6 a 35 meses. Métodos: Estudio retrospectivo en el que se aplicó una estrategia de educación alimentaria para la reducción de la anemia con evaluación pretest y postest. La educación alimentaria incluye componentes educativos, comunicacionales y tecnológicos. Se analizaron los datos correspondientes a las réplicas de la educación alimentaria en ocho regiones del Perú, de quienes cumplieron con una frecuencia de 2 veces por semana, durante dos meses. El reclutamiento de padres, madres o cuidadores (350) estuvo dirigido a quienes tenían niños de 6 a 35 meses, usuarios de un establecimiento de salud y con un diagnóstico de anemia utilizando como indicador la hemoglobina ( $\mathrm{Hb}$ ) del niño(a) al inicio y al final de la educación alimentaria. Resultados: El mayor porcentaje de niños se encuentra en el rango de 12 a 23 meses. El promedio general del diferencial de hemoglobina (evaluación final-evaluación inicial) fue de $1,07 \mathrm{~g} / \mathrm{dL} \pm 0,80 \mathrm{~g} /$ $\mathrm{dL}(\mathrm{p}<0,01)$, y se observó que el incremento era mayor mientras más alto era el nivel de anemia. Hubo diferencia entre Lima Metropolitana y las regiones $(\mathrm{p}<0,01)$, y entre algunas regiones. Conclusiones: La educación alimentaria como estrategia que incluye preparaciones de recetas ricas en hierro, mejoró el estado de dicho mineral en los niños menores de 36 meses. Se sugiere la implementación de programas de educación alimentaria de acuerdo con la disponibilidad de alimentos en cada región.
\end{abstract}

Palabras clave: anemia, educación alimentaria, estrategia, hierro

\begin{abstract}
Introduction: In Peru, although a decrease in anemia of $3.4 \%$ was observed in 2019 compared to 2018, the prevalence continues for more than a decade. The figures describe it as a severe public health problem, which affects an average of 620 thousand children at a national level. The prevalence of anemia
\end{abstract}

\footnotetext{
${ }^{1}$ Escuela de Nutrición Humana, Universidad Norbert Wiener. Lima, Perú.

${ }^{2}$ Consultor independiente

${ }^{3}$ South American Center for Education and Research in Public Health, Universidad Norbert Wiener. Lima, Perú.
} 
in children under 36 months old is higher in rural areas and in the highlands. Despite the fact that Metropolitan Lima has one of the lowest prevalence in the country (three out of every ten children suffer from anemia), it concentrates the largest number of children with anemia, with figures similar to the number of children we obtain by adding the four or five regions with the highest prevalence in the country. Objective: To assess whether the food education strategy allows a reduction in anemia in children aged six to 35 months. Methods: This is a retrospective study in which we applied a food education strategy for the reduction of anemia with pre-test and post-test evaluation. Food education includes educational, communicational and technological components. We could analyze data corresponding to the replication of food education in eight regions of Peru of those who complied with a frequency of twice a week for two months. The recruitment of fathers, mothers or caregivers (350) was aimed at those with children aged six to 35 months, users of a health facility and with a diagnosis of anemia. We used the children's hemoglobin $(\mathrm{Hb})$ as an indicator at the beginning and at the end of the food education. Results: The highest percentage of children were in the 12 to 23 months old range. The overall mean of hemoglobin differential (final assessment - initial assessment) was $1.07 \mathrm{~g} / \mathrm{dL} \pm 0.80 \mathrm{~g} / \mathrm{dL}(\mathrm{p}<0.01)$, and it was observed that the increase was greater when the level of anemia was higher. There was difference between Metropolitan Lima and regions $(\mathrm{p}<0.01)$, and difference between some regions. Conclusions: Food education as a strategy including iron-rich recipe preparations improved iron status in children under 36 months old. The implementation of food education programs according to food availability in each region is suggested.

Keywords: anemia, food and nutrition education, strategy, iron

\section{INTRODUCCIÓN}

La Organización Mundial de la Salud (OMS) indica que la anemia está presente en la tercera parte de la población mundial, de la cual la mitad corresponde a anemia por deficiencia de hierro y se estima que afecta a 1,24 billones de individuos $\left({ }^{1}\right)$. La anemia en niños y niñas en el Perú es más alta en aquellos que residen en áreas rurales (49,0\%); el área urbana tiene 12,3 puntos porcentuales menos. En el caso de los niños que viven en la zona de la sierra, la anemia alcanza el 48,8\% ( ${ }^{2}$ ). Los tipos de anemia en países en vías de desarrollo son los siguientes: anemia ferropénica y carencial (50-70\%), anemia inflamatoria (20-40\%) y anemia congénita (3-18\%) $\left({ }^{3,4}\right)$. La anemia en los niños de 6 a 35 meses reporta, para el año 2019, una prevalencia de $40,1 \%$ y afecta anualmente a unos 620000 niños a nivel nacional $\left({ }^{5}\right)$.

El rápido crecimiento de los niños los hace vulnerables, pues las necesidades se incrementan rápidamente $\left(^{6}\right)$. La anemia se ha relacionado con pobres resultados escolares y baja productividad en la adultez, lo que afecta la economía de las personas. Existe la meta de atender a los niños pequeños para desaparecer secuelas a largo plazo $\left({ }^{7}\right)$. En ese sentido, en el Perú, se han llevado a cabo intervenciones diversas para incrementar el consumo de hierro a nivel poblacional, desde la suplementación preventiva o terapéutica; la fortificación de alimentos de consumo masivo, como la harina de trigo $\left.{ }^{8}\right)$; y, recientemente, la fortificación del arroz $\left({ }^{9}\right)$, lo que incluye preparaciones de alimentos $\left({ }^{10}\right)$. La última encuesta de consumo de alimentos realizada a nivel nacional 2015-2016 reportó que, en el 2016, en promedio, con relación a los $11 \mathrm{mg}$ de hierro diario de requerimiento en el niño de 6 meses a 8 años $\left({ }^{11}\right)$, los niños de 6 a 11 meses consumen $6,2 \mathrm{mg}$ de hierro/día, los niños de 12 a 23 meses consumen $8,0 \mathrm{mg}$ de hierro/día y los niños de 24 a 35 meses consumen $8,8 \mathrm{mg} /$ día. Sin embargo, un alto porcentaje corresponde a hierro no hemínico $(83,1 \%)$ y solo el $16,9 \%$, a hierro hemínico $\left(^{12-14}\right)$.

La educación alimentaria nutricional comprende actividades de aprendizaje que facilitan la adopción voluntaria de comportamientos alimentarios para fomentar la salud y el bienestar $\left({ }^{15}\right)$. Por ello, se convierte en una herramienta imprescindible que permite reforzar los comportamientos alimentarios 
beneficiosos y modificar los que impactan negativamente en la salud del niño. Esta herramienta es útil para evitar la anemia en una edad cuya alimentación determinará lo que pasará el resto de su vida: un adecuado desarrollo físico e intelectual que influirá a largo plazo en las habilidades, el rendimiento cognitivo, la productividad laboral y los ingresos en la adultez, y un menor riesgo frente a problemas de obesidad y enfermedades crónicas, como la diabetes, la hipertensión arterial y otras $\left({ }^{16}\right)$. Estas desventajas, sumadas a lo largo del ciclo de vida, dan como resultado personas adultas que no tienen las condiciones para desplegar su máximo potencial intelectual, físico y productivo $\left({ }^{14}\right)$.

Por tanto, es oportuno evaluar si una estrategia en educación alimentaria permite una reducción de la anemia en los niños de 6 a 35 meses de edad.

\section{MÉTODOS}

\section{Diseño de estudio}

El estudio corresponde a un estudio observacional analítico de la aplicación de educación alimentaria, desarrollada durante el 2018, como estrategia para la reducción de la anemia.

\section{Población y muestra}

La población estuvo conformada por padres o madres de niños de 6 a 35 meses, de ocho regiones del Perú, usuarios de establecimientos de atención primaria de salud. La muestra fueron 384 padres de niños de 6 a 35 meses de edad, que participaron en una intervención educativa. El muestreo fue consecutivo en cada lugar de intervención, hasta completar al menos 30 sujetos (pares madre-niño) por cada investigador de campo. La intervención se realizó en ocho regiones del Perú (Amazonas, Arequipa, Callao, Cusco, Huancavelica, Ica, Lima y Moquegua).

Entre los criterios de inclusión, se incluyó al padre, madre o cuidador de un niño de 6 a 35 meses de edad con diagnóstico de anemia, que debía contar con al menos dos controles de hemoglobina (inicial y final), reportados por el centro de salud y que participan en ocho sesiones educativas. Los criterios de exclusión fueron los niños con alguna enfermedad congénita.

\section{Procedimientos}

La educación alimentaria tuvo los siguientes pasos:

a) Diseño de recetas. Las preparaciones culinarias están diseñadas para cubrir el requerimiento de los niños de 6 a 35 meses de edad. El diseño consideró preparaciones dulces ( $10 \mathrm{~g}$ de sangrecita: 1 cucharada; 4,5 mg de hierro hemínico) y preparaciones saladas (25 g de sangrecita: 1 cucharada colmada; $6,8 \mathrm{mg}$ de hierro hemínico), ambas recetas presentaron un porcentaje de adecuación del $102,7 \%$. Las dosis y el aporte de hierro por ración se basaron en la recomendación establecida por la FAO/OMS $2001\left({ }^{11}\right)$ y el Instituto de Medicina $\left.2002{ }^{(20}\right)$. Se siguió las pautas de alimentación infantil (INS-Cenan 2004) ${ }^{(21)}$ y la composición nutricional de los alimentos (INSCenan 2009) (22).

b) Capacitación. El Colegio de Nutricionistas del Perú (CNP), durante la gestión 2016-2019, realizó la convocatoria a nutricionistas de todo el Perú. En un tiempo breve, más de 200 nutricionistas presentaron su solicitud de participación. Fueron seleccionadas 50 nutricionistas; primero se capacitó a 27 nutricionistas de atención primaria del Ministerio de Salud de Lima Metropolitana y luego a 23 nutricionistas de las regiones. Como estímulo para lograr el cumplimiento de la intervención, el CNP certificó a los nutricionistas como educadores en anemia.

c) Reclutamiento. Los nutricionistas capacitados se encargaron de reclutarlos y cada nutricionista tuvo a su cargo un total 15 padres o cuidadores. Las fechas y lugares para aplicar la intervención educativa fueron de mutuo acuerdo.

d) Intervención educativa. Los padres, madres de familia o cuidadores participaron en los talleres de preparación de alimentos, dos veces por semana durante dos meses. La metodología empleada fue "aprender haciendo". La sesión educativa nutricional ofrecida a padres, madres y cuidadores duró en promedio tres horas y tuvo el siguiente orden: i) presentación, ii) motivación, iii) educación, iv) práctica de preparación de alimentos ricos en hierro, v) servido y consumo de las preparaciones elaboradas, vi) exploración y retroalimentación de aprendizajes, y vii) cierre de la sesión. En cada sesión se enseñaron aspectos sobre prevención 
de anemia basados en la exposición dialogada participativa y el aporte de hierro de los alimentos, facilitadores e inhibidores del hierro, así como otros temas relacionados con la prevención de anemia y el cuidado de la salud de niños menores de 36 meses. Durante la sesión demostrativa, se puso énfasis en la cantidad, variedad y densidad, según el INS-Cenan $2009\left({ }^{23}\right)$.

e) Medida de hemoglobina. Se tomaron dos mediciones de hemoglobina en los niños. El dato inicial de hemoglobina corresponde a la última evaluación realizada en el establecimiento de salud, la segunda medida fue al término de la intervención educativa, fue tomada por el personal de los servicios de laboratorio como parte de sus controles del tratamiento de anemia (INS-Cenan 2012) ${ }^{(24)}$. El punto de corte de anemia de los niños se basó en los criterios de la Organización Mundial de la Salud, tanto para la medición inicial y final (sin anemia $\geq 11,0 \mathrm{mg} / \mathrm{dL}$; anemia leve $<11,0 \mathrm{mg} /$ $\mathrm{dL}$; anemia moderada $<10 \mathrm{mg} / \mathrm{dL}$; anemia severa $<7,0 \mathrm{mg} / \mathrm{dL})\left({ }^{11}\right)$. Se calculó, además, el diferencial de hemoglobina entre mediciones.

\section{Variables usadas en el estudio}

Anemia pretest y postest en niños menores de tres años.

\section{Procesamiento y análisis estadístico}

Los registros ingresaron a una base en Excel. Se empleó el programa SPSS versión 25 para el análisis, a través de la prueba de chi cuadrado, para evaluar la asociación de la anemia con el grupo de edad, sexo y región. Se empleó la prueba T de Student para datos no pareados a fin de comparar la hemoglobina y el diferencial de hemoglobina según sexo y región, y la prueba de análisis de varianza de un factor para comparar la hemoglobina y el diferencial según el grupo de edad. Asimismo, se empleó la prueba $\mathrm{T}$ de student para datos pareados, para comparar los valores de hemoglobina antes y después de la intervención. Para todos los casos, se empleó un nivel de significancia $\alpha=0,05$.

\section{Aspectos éticos}

El presente proyecto utilizó los datos del centro de referencia de la empresa Wawa Food, obtenidos durante el periodo de trabajo. Los padres, madres o cuidadores que recibieron la capacitación para preparación de dietas, no brindaron datos que permitieran identificarlos; se usaron códigos para mantener la confidencialidad y fueron de acceso exclusivo para los investigadores. Previamente, explicaron cuáles serían los pasos de la intervención $y$, si decidieron participar, firmaron una hoja de consentimiento informado. El estudio cuenta con la aprobación del comité de ética de la Universidad Norbert Wiener: Exp. N. ${ }^{\circ}$ 606-2021.

\section{RESULTADOS}

De un total de 384 niños reclutados para la intervención, 350 llegaron a tener las dos muestras de hemoglobina y sus padres, madres o cuidadores participaron en al menos la mitad de las capacitaciones. De ellos, los niños de 6 a 11 meses fueron 106 (52,8\% fueron hombres y 47,2\% fueron mujeres), de 12 a 23 meses fueron $150(58,7 \%$ varones y $41,3 \%$ mujeres), y de 24 a 36 meses 94

Tabla 1. Características sociodemográficas de los niños en la intervención

\begin{tabular}{|c|c|c|}
\hline Características & Hombre (\%) & Mujer (\%) \\
\hline \multicolumn{3}{|l|}{ Edad agrupada } \\
\hline 6 a 11 meses $(n=106)$ & 52,8 & 47,2 \\
\hline 12 a 23 meses $(n=150)$ & 58,7 & 41,3 \\
\hline 24 a 35 meses $(n=94)$ & 54,3 & 45,7 \\
\hline \multicolumn{3}{|l|}{ Región } \\
\hline Amazonas ( $n=15)$ & 46,2 & 53,8 \\
\hline Arequipa $(n=45)$ & 53,3 & 46,7 \\
\hline Callao $(n=26)$ & 50,0 & 50,0 \\
\hline Cusco $(n=94)$ & 56,4 & 43,6 \\
\hline Huancavelica $(n=20)$ & 80,0 & 20,0 \\
\hline Ica $(n=12)$ & 50,0 & 50,0 \\
\hline Lima Metropolitana $(n=78)$ & 55,1 & 44,9 \\
\hline Moquegua $(n=62)$ & 54,8 & 45,2 \\
\hline \multicolumn{3}{|l|}{ Región Agrupada } \\
\hline Lima Metropolitana $(n=104)$ & 53,8 & 46,2 \\
\hline Regiones $(n=246)$ & 56,5 & 43,5 \\
\hline \multicolumn{3}{|l|}{ Altitud } \\
\hline \multicolumn{3}{|l|}{ De 0 a 999 m s. n. m. $(n=146)$} \\
\hline \multicolumn{3}{|l|}{1000 a 1999 m s. n. m. $(n=75)$} \\
\hline \multicolumn{3}{|l|}{2000 a más m s. n. m. $(n=129)$} \\
\hline Total $(n=350)$ & 55,7 & 44,3 \\
\hline
\end{tabular}


(54,3\% varones y $45,7 \%$ mujeres). Para todos los subgrupos de edad, la proporción de hombres $(55,7 \%)$ fue superior a la de mujeres $(44,3 \%)$.

Las regiones con mayor proporción de niñas y niños participantes fueron Cusco (26,9\%), Lima $(22,3 \%)$, Moquegua (17,7\%), Arequipa (12,9\%), Callao $(7,4 \%)$, Huancavelica (5,7\%), Amazonas (3,7\%) e Ica $(3,4 \%)$. La gran mayoría de las niñas y los niños fueron de las regiones (70,3\%), en comparación con Lima Metropolitana $(29,7 \%)$.

En la evaluación inicial, el 93,7\% de los niños participantes de la intervención tuvo diagnóstico de anemia y el restante $6,3 \%$, valores de hemoglobina menores de 11,5 g/dL. El grupo de 24 a 35 meses tuvo una menor proporción de anemia, aunque esta no fue estadísticamente significativa $(\mathrm{p}>0,05)$. No hubo asociación entre la anemia y el sexo, la región, la región agrupada ni la altitud. La hemoglobina inicial fue de 10,2 $\pm 0,74 \mathrm{mg} / \mathrm{dL}$.

En la evaluación final, el 22,9\% de los niños participantes de la intervención tuvo diagnóstico de anemia. El grupo de 6 a 11 meses tuvo una significativa mayor proporción de anemia que los otros grupos de edad ( $\mathrm{p}<0,01)$. No hubo asociación entre la anemia y el sexo, la región ni la región agrupada, pero sí con la altitud $(\mathrm{p}<0,05)$, con un nivel de anemia menor al presentado en el resto de las regiones $(p<0,05)$. La hemoglobina final fue de $11,4 \pm 0,77 \mathrm{mg} / \mathrm{dL}$.

Tabla 2. Porcentaje de anemia al inicio y final de la intervención, según características sociodemográficas

\begin{tabular}{|c|c|c|c|c|}
\hline \multirow{2}{*}{ Características } & \multicolumn{2}{|c|}{ Inicio } & \multicolumn{2}{|c|}{ Final } \\
\hline & Normal & Anemia & Normal & Anemia \\
\hline Total & 6,30 & 93,70 & 77,10 & 22,9 \\
\hline \multicolumn{5}{|l|}{ Edad agrupada } \\
\hline 6 a 11 meses $(n=106)$ & 5,70 & 94,30 & 66,00 & 34,00 \\
\hline 12 a 23 meses $(n=150)$ & 4,00 & 96,00 & 81,30 & 18,70 \\
\hline 24 a 35 meses $(n=94)$ & 10,60 & 89,40 & 83,00 & 17,00 \\
\hline \multicolumn{5}{|l|}{ Región } \\
\hline Amazonas $(n=15)$ & 0,00 & 100,00 & 76,90 & 23,10 \\
\hline Arequipa $(n=45)$ & 0,00 & 93,30 & 75,60 & 24,40 \\
\hline Callao $(n=26)$ & 3,80 & 96,20 & 73,10 & 26,90 \\
\hline Cusco $(n=94)$ & 3,20 & 96,80 & 75,50 & 24,50 \\
\hline Huancavelica $(n=20)$ & 5,00 & 95,00 & 50,00 & 50,00 \\
\hline Ica $(n=12)$ & 0,00 & 100,00 & 83,30 & 16,70 \\
\hline Lima Metropolitana $(\mathrm{n}=78)$ & 9,00 & 91,00 & 79,50 & 20,50 \\
\hline Moquegua $(n=62)$ & 11,30 & 88,70 & 87,10 & 12,90 \\
\hline \multicolumn{5}{|l|}{ Región agrupada } \\
\hline Lima Metropolitana $(n=104)$ & 7,70 & 92,30 & 77,90 & 22,10 \\
\hline Regiones $(n=246)$ & 5,70 & 94,30 & 76,80 & 23,20 \\
\hline \multicolumn{5}{|l|}{ Sexo } \\
\hline Hombre $(n=195)$ & 5,60 & 94,40 & 77,40 & 22,60 \\
\hline Mujer $(n=155)$ & 7,10 & 92,90 & 76,80 & 23,20 \\
\hline \multicolumn{5}{|l|}{ Altitud } \\
\hline De 0 a 999 m s. n. m. $(n=146)$ & 5,50 & 94,50 & 78,80 & 21,20 \\
\hline 1000 a 1999 m s. n. m. $(n=75)$ & 9,30 & 90,70 & 85,30 & 14,70 \\
\hline 2000 a más m s. n. m. $(n=129)$ & 5,40 & 94,60 & 70,50 & 29,50 \\
\hline Total $(n=350)$ & 6,30 & 93,70 & 77,10 & 22,90 \\
\hline
\end{tabular}

Fuente: base de datos

Elaboración: propia

${ }^{*} p<0,01{ }^{* *} p<0,05$ 
Eficacia en la prevención y recuperación de anemia

Entre la evaluación inicial y la final puede observarse una reducción de la anemia de aproximadamente 70 puntos porcentuales $(\mathrm{p}<0,01)$. Según el nivel de anemia al inicio de la intervención, en el grupo de niños con anemia leve, el porcentaje de recuperación fue casi del $90 \%$. Para el caso de los niños con anemia moderada, el porcentaje de recuperación fue casi del $50 \%$. Además, del grupo que inició sin anemia, casi todos se mantuvieron sin anemia (90,5\%). La intervención tuvo una alta eficacia en recuperación de la anemia y en su prevención.

\section{Incremento de hemoglobina}

La diferencia de hemoglobina entre la evaluación inicial y la final fue estadísticamente significativa $(\mathrm{p}<0,01)$. El promedio general del diferencial de hemoglobina (evaluación final-evaluación inicial) fue de $1,07 \mathrm{~g} / \mathrm{dL} \pm 0,80 \mathrm{~g} / \mathrm{dL}$. Se observó una diferencia significativa según el diagnóstico inicial de anemia ( $<<0,01)$, y se observó que el incremento era mayor mientras mayor era el nivel de anemia. Se halló también una diferencia entre Lima Metropolitana y las regiones $(\mathrm{p}<0,01)$, y diferencias entre algunas regiones.

\section{DISCUSIÓN}

La intervención se aplicó a 350 padres, madres o cuidadores, quienes al menos tuvieron seis sesiones para aprender recetas ricas en hierro. En promedio, todos los niños tuvieron dos mediciones de hemoglobina. En todas las regiones, se tuvo más padres, madres o cuidadoras de niños que niñas. Las

Tabla 3. Diagnóstico final de anemia, según diagnóstico inicial

Diagnóstico final de anemia

\begin{tabular}{lccc}
\hline Diagnóstico inicial de anemia & Normal & Anemia leve & Anemia moderada \\
\hline Normal $(n=21)$ & $90,5 \%$ & $9,5 \%$ & $0,0 \%$ \\
\hline Anemia leve $(n=225)$ & $88,0 \%$ & $11,6 \%$ & $0,4 \%$ \\
\hline Anemia moderada $(n=104)$ & $51,0 \%$ & $40,4 \%$ & $8,7 \%$ \\
\hline Total $(n=350)$ & $77,1 \%$ & $20,0 \%$ & $2,9 \%$ \\
\hline
\end{tabular}

Fuente: base de datos

Elaboración: propia regiones con mayor población en la intervención fueron Cusco, Lima y Moquegua (26,9\%, 22,3\% y $17,7 \%$, respectivamente). Entre los valores de anemia iniciales y finales se halló diferencia ( $\mathrm{p}<$ $0,01)$. El promedio diferencial de hemoglobina fue de $1,07 \mathrm{~g} / \mathrm{dL} \pm 0,80 \mathrm{~g} / \mathrm{dL}$, valor $\mathrm{p}<0,01$, y se observó que el incremento fue mayor mientras mayor fue el nivel de anemia. Lima Metropolitana es diferente a las demás regiones $(\mathrm{p}<0,01)$.

Tabla 4. Incremento de hemoglobina, según características sociodemográficas.

\begin{tabular}{|c|c|c|}
\hline Características & Promedio & $\mathrm{DE}$ \\
\hline \multicolumn{3}{|l|}{ Diagnóstico inicial de anemia* } \\
\hline Normal $(\mathrm{n}=21)^{* *}$ & 0,60 & 0,70 \\
\hline Anemia leve $(n=225)^{* *}$ & 0,94 & 0,70 \\
\hline Anemia moderada $(n=104)$ & 1,43 & 0,90 \\
\hline \multicolumn{3}{|l|}{ Edad agrupada } \\
\hline 6 a 11 meses $(n=106)$ & 1,00 & 0,74 \\
\hline 12 a 23 meses $(n=150)$ & 1,17 & 0,87 \\
\hline 24 a 35 meses $(n=94)$ & 0,97 & 0,73 \\
\hline \multicolumn{3}{|l|}{ Región*** } \\
\hline Amazonas $(n=15)$ & 1,42 & 0,79 \\
\hline Arequipa $(n=45)$ & 0,95 & 0,73 \\
\hline Callao $(n=26)$ & 0,88 & 0,48 \\
\hline Cusco $(n=94)$ & 1,23 & 0,84 \\
\hline Huancavelica $(n=20)$ & 0,48 & 0,63 \\
\hline Ica $(n=12)$ & 2,08 & 0,70 \\
\hline Lima Metropolitana $(n=78)$ & 0,95 & 0,69 \\
\hline Moquegua $(\mathrm{n}=62)$ & 1,05 & 0,85 \\
\hline \multicolumn{3}{|l|}{ Región Agrupada* } \\
\hline Lima Metropolitana $(n=104)$ & 0,93 & 0,64 \\
\hline Regiones $(n=246)$ & 1,12 & 0,85 \\
\hline \multicolumn{3}{|l|}{ Sexo } \\
\hline Hombre $(n=195)$ & 1,09 & 0,85 \\
\hline Mujer $(n=155)$ & 1,03 & 0,74 \\
\hline \multicolumn{3}{|l|}{ Altitud } \\
\hline De 0 a 999 m s. n. m. $(n=146)$ & 1,03 & 0,74 \\
\hline 1000 a 1999 m s. n. m. $(n=75)$ & 1,12 & 0,85 \\
\hline 2000 a más m s. n. m. $(\mathrm{n}=129)$ & 1,08 & 0,85 \\
\hline Total $(n=350)$ & 1,07 & 0,80 \\
\hline \multicolumn{3}{|c|}{$\begin{array}{l}\text { Fuente: base de datos } \\
\text { Elaboración: propia } \\
{ }^{*} p<0,01{ }^{* *} p<0.05 \text { (normal versus anemia leve) } \\
{ }^{* * *} \text { (Ica, Lima Metropolitana y Arequipa no son diferentes entre sí; Lima } \\
\text { Metropolitana y Callao no son diferentes de Moquegua; Callao no es } \\
\text { diferente de Huancavelica; Cusco Amazonas y Moquegua no son diferentes } \\
\text { entre sí; Arequipa no es diferente de Moquegua; Moquegua solo es diferente } \\
\text { de Ica y Huancavelica). }\end{array}$} \\
\hline
\end{tabular}


El estudio de Fançony et al. brindó información sobre recetas, calidad nutricional de dietas, diversidad de alimentos, frecuencia mínima de comidas e incremento de ingesta de ácido fólico, hierro y vitamina A. Se realizó un seguimiento de 12 meses y un total de seis sesiones entregadas a los cuidadores o madres de niños menores de cinco años, aunque no se trató solo el aspecto nutricional, también se abordaron aspectos como desparasitación y limpieza. Se encontró una disminución de la anemia que va del $82,0 \%$ al $55,4 \%$, mientras que, en nuestro estudio en niños de 6 a 11 meses, la reducción fue del $94,30 \%$ al $34,00 \%$; en los niños de 12 a 23 meses, del $96,00 \%$ al $18,70 \%$; y, finalmente, en el grupo de 24 a 35 meses, del $89,40 \%$ al $17,00 \%\left({ }^{25}\right)$.

En nuestro estudio, el promedio diferencial de hemoglobina fue de $1,07 \mathrm{~g} / \mathrm{dL} \pm 0,80 \mathrm{~g} / \mathrm{dL}, \mathrm{p}<$ 0,01. Autores como Sunuwar et al., en el año 2019, educaron a madres durante 10 semanas para que mejoraran su score en anemia y alimentos ricos en hierro, y encontraron diferencias entre los casos y controles $[8,26 \pm 4,57$ vs. $1,05 \pm 6,59, \mathrm{p}<0,001]$ ${ }^{(26)}$. En ese sentido, Penny et al. trabajaron con madres de 187 niños entre 6 y 18 meses en una zona periurbana del Perú, y encontraron un pobre consumo de hierro del tipo hemínico, con valores menores a los recomendados $\left({ }^{27}\right)$. Esa es una realidad que también aparece en las ocho regiones donde se desarrolló nuestro estudio, dado el elevado número de casos de anemia. La falta de conocimiento y las prácticas nutricionales inapropiadas por parte de los cuidadores son una causa importante de una dieta deficiente en hierro $\left({ }^{28}\right)$. Bilenko, en Israel, encontró que la presencia de anemia ferropénica en los lactantes está afectada inversamente por el nivel de conocimiento materno sobre la anemia y la adherencia a la suplementación con hierro; halló también que el bajo nivel de conocimiento está directamente relacionado con la baja adherencia $\left({ }^{29}\right)$.

Todas las madres de este estudio recibieron en promedio seis sesiones, en las que aprendieron a elaborar preparaciones ricas en hierro. En ese sentido, la metodología "aprender haciendo" (learning by doing) establece que los contenidos teóricos son necesarios para avanzar en el aprendizaje de cualquier área de conocimiento, pero para lograr retenerlos y dotarlos de utilidad es mejor experimentar con ellos, de modo que el proceso persista en el tiempo. Autores como Inayati et al. ofrecieron a madres de niños entre 6 y 60 meses intervenciones que duraron una semana, $y$ sus resultados mostraron que la educación intensiva semanal en nutrición fue significativa (del $62,3 \%$ al $37,7 \%$; $<0,001)\left({ }^{30}\right)$.

Entre las limitaciones de esta intervención se tuvo la falta de disponibilidad local de alimentos ricos en hierro, lo que limitó la diversificación de la dieta. No se ha podido probar si la madre buscó otros mecanismos para garantizar fuentes de hierro, tampoco se conocen precios de los productos. Otra de nuestras limitaciones se refiere a las características sociodemográficas de la madre o cuidadora y a la apreciación de los talleres.

\section{CONCLUSIONES}

$\mathrm{Al}$ inicio de la intervención, el 93,7\% de los niños tenía anemia y al final de la misma, solo el 23,9\%. Casi todos los niños con diagnóstico inicial anemia leve y la mitad de los niños con anemia moderada se recuperaron. Hubo un mayor incremento de hemoglobina mientras mayor fue el nivel de anemia, y fue mayor el incremento de hemoglobina en las regiones en comparación con Lima Metropolitana. Debido a las causas multifactoriales de esta enfermedad, determinar la eficacia de esta intervención puede promover estudios para aplicar estrategias que reduzcan la anemia.

\section{AGRADECIMIENTO}

Agradecemos a todas las autoridades de los establecimientos de salud donde se desarrollaron las intervenciones educativas, por permitirnos trabajar en los locales. Asimismo, a todo el equipo de nutricionistas que fueron seleccionados para llevar a cabo este proyecto.

\section{CONTRIBUCIÓN DE LOS AUTORES}

Rosa Elena Cruz, Iván Carbajal, Saby Mauricio Alza y Michelle Fátima Lozada-Urbano son autores y responsables de la redacción, revisión y aprobación de la versión final del artículo. 


\section{POTENCIALES CONFLICTOS DE INTERESES}

Ninguno.

\section{FINANCIAMIENTO}

Este trabajo fue autofinanciado. Agradecemos a la empresa Wawa Food, que hizo una donación de empaques de sangrecita de cerdo para la preparación de recetas en la ciudad de Lima.

\section{REFERENCIAS BIBLIOGRÁFICAS}

1. Vos T, Abajobir AA, Abate KH, Abbafati C, Abbas K M, Abd-Allah F, Abera, SF. Global, regional, and national incidence, prevalence, and years lived with disability for 328 diseases and injuries for 195 countries, 1990-2016: a systematic analysis for the Global Burden of Disease Study 2016 [published correction appears in Lancet. 2017 Oct 28; 390 (10106): e38]. Lancet. 2017; 390(10100): 1211-59. doi:10.1016/S0140-6736(17)32154-2

2. Instituto Nacional de Estadística e Informática. Encuesta Demográfica y de Salud Familiar 2019 Nacional y Regional (Endes 2019) [Internet]. Lima: INEI; 2020. Disponible en: https://www.inei.gob. pe/media/MenuRecursivo/publicaciones_digitales/ Est/Endes2019/

3. Instituto Nacional de Estadística e Informática. Encuesta Demográfica y de Salud Familiar 2016 Nacional y Regional (Endes 2016) [Internet]. Lima: INEI; 2017. Disponible en: http://proyectos.inei. gob.pe/endes/resultados.asp

4. Kassebaum NJ, Jasrasaria R, Naghavi M, et al. A systematic analysis of global anemia burden from 1990 to 2010. Blood. 2014; 123(5): 615-24. doi:10.1182/blood-2013-06-508325

5. Instituto Nacional de Estadística e Informática. Indicadores de Resultados de los Programas Presupuestales, 2014-2019. Encuesta Demográfica y de Salud Familiar. Lima: INEI; 2020.

6. Rastogi T, Mathers C. Global burden of Iron Deficiency Anaemia in the year 2000 [Internet]. 2002 [cited 2020 sep 18]. Disponible en: https:// www.who.int/healthinfo/statistics/bod irondeficiencyanaemia.pdf

7. Zavaleta N, Astete L. Efecto de la anemia en el desarrollo infantil: consecuencias a largo plazo. Rev Peru Med Exp Salud Pública. 2017; 34(4): 716-22.
8. Instituto Nacional de Salud - Centro Nacional de Alimentación y Nutrición. Control analítico de la fortificación de la harina de trigo en los molinos del Perú año 2012. Verificación de la fortificación en molinos del Perú, año 2012. Disponibilidad, acceso y consumo de harina de trigo y sus derivados, en el Perú, año 2011. Lima: INS, CENAN; 2017.

9. Ministerio de Salud del Perú. Aprueban fichas de homologación de "Arroz fortificado grado extra", "Arroz fortificado grado superior" y "Arroz fortificado grado corriente" Resolución Ministerial N. ${ }^{\circ}$ 745-2018/MINSA. Lima; 2018.

10. Presidencia del Consejo de Ministros. Aprueba el Plan Multisectorial de lucha contra la anemia. Decreto Supremo N. ${ }^{\circ}$ 68-2018-PCM. Lima; 2018.

11. Food and Agriculture Organization of the United Nations, World Health Organization. Human Vitamin and Mineral Requirements. Roma: FAO/ WHO; 2001.

12. Instituto Nacional de Salud. Informe preliminar Encuesta de Vigilancia Alimentaria y Nutricional por Etapas de Vida (VIANEV). Niños menores de 36 meses. Lima; 2016.

13. Guillén-López S, Vela-Amieva M. Desventajas de la introducción de la leche de vaca en el primer año de vida. Acta Pediatr Mex 2010; 31(3): 123-8.

14. Svarch E. Iron deficiency anemia in the infant. Rev Cubana Pediatr. 2015; 87(4): 395-8.

15. Puszko B, Sánchez S, et al. El impacto de la educación alimentaria nutricional en el embarazo: una revisión de las experiencias de intervención. Revista Chilena de Nutrición. 2017; 44(1). http://dx.doi.org/10.4067/ S0717-75182017000100011 
16. Martínez-Villegas O, Baptista H, González A. Anemia por deficiencia de hierro en niños: un problema de salud nacional. Revista de Hematología Mex. 2019; 20(2): 96-105.

17. Robles Mena H. Influencia de las intervenciones del plan nacional de lucha contra la anemia en la condición de anemia en menores de tres años, Cusco, 2018 [Tesis doctoral]. Universidad César Vallejo; 2019.

18. Castro Chamorro S. Programa Qali Warma y anemia ferropénica en niños menores de 5 años de Chaupimarca-Pasco, 2019 [Tesis de maestría]. Universidad César Vallejo; 2020.

19.Sandoval Zavaleta C. Impacto del plan de intervenciones sectoriales en la modificación de conocimiento en madres y reducción de anemia en niños menores de 5 años [Tesis de maestría]. Universidad César Vallejo; 2019.

20. Food and Nutrition Board, Institute of Medicine. Dietary Reference Intakes (DRI) for vitamin A, vitamin $\mathrm{K}$, arsenic, boron, chromiun, copper, iodine, iron, manganese, molybdenum, nickel, silicon, and, vanadium. Institute of Medicine of the National Academies. Washington DC: The Nacional Academy Press; 2002. Disponible en: https://pubmed.ncbi. nlm.nih.gov/25057538/

21. Instituto Nacional de Salud, Centro Nacional de Alimentación y Nutrición. Lineamientos de Nutrición Materno Infantil del Perú. Lima: INSCENAN; 2004. Disponible en:https://repositorio.ins. gob.pe/bitstream/handle/INS/218/CENAN0060. pdf? sequence $=1 \& \% 0$ AsAllowed $=\mathrm{y} \% 0 \mathrm{~A}$

22. Instituto Nacional de Salud, Centro Nacional de Alimentación y Nutrición. Tablas Peruanas de Composición de Alimentos. Lima: INS-CENAN; 2009. Disponible en: https://www.ins.gob.pe/ insvirtual/images/otrpubs/pdf/Tabla \%20de\%20 Alimentos.pdf

23. Centro Nacional de Alimentación y Nutrición. Documento Técnico: Procedimiento para el diseño y validación de recetas nutricionales (2012). Dirección General de CENAN. Disponible en: https://goo.su/3KCANGm
24.Jordan T. Guía Técnica. Procedimiento para la determinación de la hemoglobina mediante hemoglobinómetro portátil. Instituto Nacional de Salud. Disponible en: http://repositorio.ins.gob.pe/ handle/INS/226

25. Fançony C, Soares Â, Lavinha J, Barros H, Brito M. Efficacy of Nutrition and WASH/Malaria Educational Community-Based Interventions in Reducing Anemia in Preschool Children from Bengo, Angola: Study Protocol of a Randomized Controlled Trial. Int J Environ Res Public Health. 2019; 16(3): 466.

26. Sunuwar DR, Sangroula RK, Shakya NS, Yadav R, Chaudhary NK, Pradhan PMS. Effect of nutrition education on hemoglobin level in pregnant women: A quasi-experimental study. PLoS One. 2019; 14(3): e0213982.

27.Penny ME, Creed-Kanashiro HM, Robert RC, Narro MR, Caulfield LE, Black RE. Effectiveness of an educational intervention delivered through the health services to improve nutrition in young children: a cluster-randomised controlled trial. Lancet. 2005; 365(9474): 1863-72.

28. Collings R, Harvey LJ, Hooper L, et al. The absorption of iron from whole diets: a systematic review. Am J Clin Nutr. 2013; 98(1): 65-81. doi:10.3945/ ajcn.112.050609

29. Bilenko N, Yehiel M, Inbar Y, Gazala E. The association between anemia in infants, and maternal knowledge and adherence to iron supplementation in southern Israel. Isr Med Assoc J. 2007; 9(7): 521-4.

30. Inayati DA, Scherbaum V, Purwestri RC, et al. Combined intensive nutrition education and micronutrient powder supplementation improved nutritional status of mildly wasted children on Nias Island, Indonesia. Asia Pac J Clin Nutr. 2012; 21(3): 361-73. 